\title{
Reduced Neuronal Transcription of Escargot, the Drosophila Gene Encoding a Snail-Type Transcription Factor, Promotes Longevity
}

OPEN ACCESS

Edited by:

John Tower,

University of Southern California,

United States

Reviewed by:

Jie Shen,

Hangzhou Dianzi University, China

Svetlana Radyuk,

Southern Methodist University,

United States

*Correspondence:

Elena G. Pasyukova

egpas@rambler.ru

†These authors have contributed equally to this work.

Specialty section:

This article was submitted to

Genetics of Aging,

a section of the journal

Frontiers in Genetics

Received: 31 October 2017 Accepted: 12 April 2018 Published: 30 April 2018

Citation:

Symonenko AV, Roshina NV Krementsova AV and Pasyukova EG

(2018) Reduced Neuronal Transcription of Escargot, the

Drosophila Gene Encoding a

Snail-Type Transcription Factor,

Promotes Longevity.

Front. Genet. 9:151.

doi: 10.3389/fgene.2018.00151

\author{
Alexander V. Symonenko ${ }^{1 \dagger}$, Natalia V. Roshina ${ }^{1,2 \dagger}$, Anna V. Krementsova ${ }^{1,3}$ and \\ Elena G. Pasyukova ${ }^{1 *}$ \\ ${ }^{1}$ Laboratory of Genome Variation, Institute of Molecular Genetics, Russian Academy of Sciences, Moscow, Russia, \\ ${ }^{2}$ Laboratory of Genetic Basis of Biodiversity, N. I. Vavilov Institute of General Genetics, Russian Academy of Sciences, \\ Moscow, Russia, ${ }^{3}$ Laboratory of Kinetics and Mechanisms of Enzymatic and Catalytic Reactions, N. M. Emanuel Institute of \\ Biochemical Physics, Russian Academy of Sciences, Moscow, Russia
}

In recent years, several genes involved in complex neuron specification networks have been shown to control life span. However, information on these genes is scattered, and studies to discover new neuronal genes and gene cascades contributing to life span control are needed, especially because of the recognized role of the nervous system in governing homeostasis, aging, and longevity. Previously, we demonstrated that several genes that encode RNA polymerase II transcription factors and that are involved in the development of the nervous system affect life span in Drosophila melanogaster. Among other genes, escargot (esg) was demonstrated to be causally associated with an increase in the life span of male flies. Here, we present new data on the role of esg in life span control. We show that esg affects the life spans of both mated and unmated males and females to varying degrees. By analyzing the survival and locomotion of the esg mutants, we demonstrate that esg is involved in the control of aging. We show that increased longevity is caused by decreased esg transcription. In particular, we demonstrate that esg knockdown in the nervous system increased life span, directly establishing the involvement of the neuronal esg function in life span control. Our data invite attention to the mechanisms regulating the esg transcription rate, which is changed by insertions of DNA fragments of different sizes downstream of the structural part of the gene, indicating the direction of further research. Our data agree with the previously made suggestion that alterations in gene expression during development might affect adult lifespan, due to epigenetic patterns inherited in cell lineages or predetermined during the development of the structural and functional properties of the nervous system.

Keywords: Drosophila melanogaster, life span, longevity, aging, the nervous system, transcription factor, transcription

\section{INTRODUCTION}

The nervous system is a key player in maintaining homeostasis and the structural and functional integrity of living beings and, hence, in controlling aging and longevity (Alcedo et al., 2013). The nervous system is a network of specialized neuronal cells, and their identity is established during development and maintained throughout adulthood due to the 
expression of genes coding for neurotransmitters and neuropeptides, ion channels, receptor and motor proteins, recognition and adhesion molecules, and other neuronal genes (Hobert, 2011).Given the role of the nervous system in life span control, a reasonable question would be whether genes defining the cellular specificity of neurons are also involved, in some way, in the regulation of longevity. Some of these genes have been shown to affect life span (Pasyukova et al., 2015). For example, paralytic, which encodes the major voltage-gated sodium channel and maleless, which encodes the ATP-dependent, double-stranded RNA helicase required for adenosine-to-inosine RNA editing and the proper expression of paralytic, are involved in both the regulation of synaptic activity (Reenan et al., 2000; Zhong and Wu, 2004) and in longevity control (Reenan and Rogina, 2008). A loss-of-function mutation in the Odorant receptor co-receptor gene that encodes a co-receptor protein participating in the sensory perception of smell (Mukunda et al., 2014) and the Gustatory receptor $63 a$ that encodes the gustatory receptor that participates in the detection of carbon dioxide (Kwon et al., 2007) extend longevity (Libert et al., 2007; Poon et al., 2010). However, information on the role of neuronal genes in life span control remains scarce.

We have already demonstrated that several genes that encode RNA polymerase II transcription factors and that are involved in neural development affect life span in Drosophila melanogaster (Pasyukova et al., 2004; Rybina and Pasyukova, 2010; Roshina et al., 2014; Rybina et al., 2017). Among other genes, escargot (esg) was identified as a candidate gene affecting life span in a screen of more than 1,500 insertion mutations and the insertion located downstream of esg was further confirmed to be causally associated with life span control (Magwire et al., 2010). esg (http://flybase.org/reports/FBgn0001981.html) is localized on the second chromosome, and its single exon encodes an RNA polymerase II transcription factor that possesses five $\mathrm{Zn}^{2+}$-finger DNA-binding domains and a P-DLS-K domain; it can both repress and activate transcription by binding to the consensus DNA sequence $5^{\prime}$-A/GCAGGTG-3' or to the dCtBP co-repressor (Ashraf et al., 1999; Ashraf and Ip, 2001; Cai et al., 2001). esg belongs to the Snail family of genes that are involved in the development of the nervous system in arthropods and chordates (Manzanares et al., 2001). In Drosophila melanogaster, Esg and other Snail proteins act to control asymmetric neuroblast division during embryogenesis; however, Esg functions are not exclusively neuronal, and it also participates in the maintenance of intestinal and male germ cells, regulates tracheal morphogenesis and development of the genital disk, and determines wing cell fate (http://flybase.org/reports/ FBgn0001981.html).

Here, we present new data on the role of esg in life span control. Analysis of the $e s g^{B G 01042}$ mutation allowed us to show that esg is involved in the regulation of life span, to varying degrees, in unmated and mated males and females. The esg $g^{B G 01042}$ mutation also increased locomotion, specifically during old age, indicating that the mutation slowed down aging. The increase in longevity was caused by decreased esg transcription associated with structural changes in the DNA sequences downstream of the gene. This result was corroborated by the fact that the decrease in the esg transcription rate due to the gene-specific RNAi knockdown in the nervous system increased life span, directly establishing the involvement of the neuronal function of esg in life span control.

\section{MATERIALS AND METHODS}

\section{Fly Strains and Crosses}

The w[1118]; P\{GT1\}esg $g^{B G 01042}$ (esgP) line is a homozygous Drosophila melanogaster line with a $8.5 \mathrm{~kb} P\{G T 1\}$ insertion located 602 bp downstream of the esg gene in a $w^{1118}(F)$ (Control M) line background (http://flypush.imgen.bcm.tmc.edu/pscreen/ transposons.html; Bellen et al., 2004; see also Magwire et al., 2010). Both lines were obtained from Trudy Mackay (North Carolina State University, USA).

The $w^{1118} ; P\{G D 1437\} v 9793(\mathrm{Kdw} 1)$ line (estimated off-target effect is 4\%; http://www.genomernai.org/v17/reagentdetails/ 9793) was used to provide esg RNAi knockdown; knockdown; the $w^{1118}$ (Control K1) line without a transgene providing RNAi was used as a control line for the esg RNAi knockdown, as suggested by the manufacturer. Both lines were produced by and obtained from Vienna Drosophila Resource Center (Dietzl et al., 2007; http://stockcenter.vdrc.at/control/main).

Several lines were obtained from the Bloomington Drosophila Stock Center (USA) (http://flystocks.bio.indiana.edu/).

The $y^{1} \quad v^{1} ; \quad$ PTRiP.JF03134\}attP2 (Kdw2) and $y^{1} v^{1}$; P\{TRiP.HMS00025\}attP2 (Kdw3) lines were used to provide esg RNAi knockdown (estimated off-target effects are 0\%; http:// www.genomernai.org/v17/reagentdetails/DRSC03530; http:// www.genomernai.org/v17/reagentdetails/DRSC37545); the $y^{1}$ $v^{1} ; P\left\{y^{+t 7.7}=\right.$ CaryP $\}$ attP2 (Control K2) line without a transgene providing RNAi was used as a control line for esg RNAi knockdown, as suggested by the manufacturer (http://flystocks. bio.indiana.edu/Browse/TRiPtb.htm).

The $P\left\{w^{+m W . h s}=\right.$ GawB $\}$ elav ${ }^{C 155} w^{1118} ; P\left\{w^{+m C}=U A S\right.$ $D c r-2 . D\} 2$ line was used to induce the expression of transgenic constructs in the nervous system. This driver line proved to be effective in our previous work, according to the real time RT-qPCR data (Rybina et al., 2017; Symonenko, unpublished results).

To induce expression of transgenic constructs, females of the driver line were crossed to males of Control K1, Control $\mathrm{K} 2, \mathrm{Kdw} 1, \mathrm{Kdw} 2$, and Kdw3 lines. Hybrid progeny used for life span measurements were further denoted as: Control K1, Control K2, Kdw1, Kdw2, and Kdw3. Life spans of hybrid Kdw1 individuals were compared to life spans of hybrid Control K1 individuals; life spans of hybrid Kdw2 and Kdw3 individuals were compared to life spans of hybrid Control K2 individuals. Dicer was present in all, both Control and Kdw, individuals.

Flies were kept at $25^{\circ} \mathrm{C}$ on a medium of semolina, sugar, raisins, yeast and agar with nipagin, propionic acid and streptomycin. For all experiments, the flies were collected from cultures with moderately controlled density: in each vial, 10-15 fertilized females of approximately the same age (5-20 days old) were allowed to lay eggs for 4 days. 


\section{Tests for Wolbachia}

Prior to the experiments, all the lines were checked for the presence of Wolbachia, a Drosophila symbiont known to affect life history traits (McGraw and O'Neill, 2004), via quantitative PCR (MiniOpticon real-time PCR detection system, Bio-Rad) with primers for the $16 \mathrm{~S}$ rRNA gene, 5'-CATACCTATTCGAAGGGATAG-3', and 5'-AGCTTCGAGTGAAACCAATTC-3' (Werren and Windsor, 2000). Negative results were obtained for all lines except Control $\mathrm{K} 1$. This line was treated with tetracycline $(0.25 \mathrm{mg} / \mathrm{mL}$, Holden et al., 1993) for three generations followed by at least three generations of recovery, before it was used in experiments.

\section{PCR and Sequencing}

DNA was extracted from batches of 20 flies of each genotype using a standard phenol-chloroform procedure (Sambrook et al., 1989). DNA was used in PCR reactions with esg1 $5^{\prime}$ AGTCAATTCCTATTTCCGGC $3^{\prime}$ and esg2 $5^{\prime}$ CACCCGAACGATACCTTACC 3' primers (expected product size of $508 \mathrm{bp}$ ). PCR products were sequenced on an ABI PRIZM 310 Genetic Analyzer (Applied Biosystems) using the esg1 and esg2 primers and a Big Dye Terminator V. 3.1. Kit (Applied Biosystems), according to the manufacturer's protocol.

\section{Life Span Assays}

Life span was measured as described by Roshina et al. (2014). To measure the life span of unmated flies, 5 virgin flies of the same genotype and sex, all collected on the same day from cultures with moderate density, were placed in replicate vials. To measure the life span of mated flies, 3 virgin males and 3 virgin females of the same genotype were placed together in replicate vials. Flies were transferred to vials with fresh food containing approximately $5 \mathrm{~mL}$ of standard medium without live yeast on the surface weekly (virgin flies) or two times a week (mated flies). The number of dead flies was recorded daily. Experiments comparing fly life spans were conducted simultaneously. Sample sizes were 49-150 flies per sex per genotype. The experiments that showed noteworthy results were repeated two to five times. Five experiments with unmated flies were done for five consecutive years. Life spans of mated males and females were measured twice with an interval of approximately 6 months. Life spans of flies with esg knockdown were measured twice for two consecutive years. The life span for each fly was estimated as the number of days alive from the day of eclosion to the day of death. Mean and median life span and survival curves were primarily used to characterize life span.

\section{Locomotion Assays}

Locomotion was measured as described by Roshina et al. (2014). Flies were collected and maintained by the same procedures as for the life span assays but without recording the deaths. Locomotion was measured, at the same time each day, in unmated males and females and in mated males at age 1, 10, 20,30,40, or 50 days. Experiments comparing locomotion were conducted simultaneously. Sample sizes were 33-100 flies (11-20 vials) per genotype per age. One day before the measurements, five virgin flies of the same sex, age and genotype or three mated flies of the same sex, age and genotype were placed in the replicate vials. After every measurement, the mated males were returned to the vials with the females. To measure locomotor activity, the vials were placed horizontally in a Drosophila Population Monitor (TriKinetics). Fly movement along the walls or in the middle of the vial crossed the infrared beam rings along the length of the vial. Beam interruptions were detected and totals were reported every $5 \mathrm{~min}$ to the host computer. Two measurements for $5 \mathrm{~min}$ were made for each vial. Locomotion was characterized as the mean number of beam interruptions per vial.

\section{Real-Time RT-qPCR}

Total RNA for real-time reverse transcription quantitative PCR (RT-qPCR) was extracted from batches of 20 whole bodies of 1 -, 10-, and 20-day-old virgin males, each batch collected from cohorts of different progeny, and from 50 embryos aged 14$20 \mathrm{~h}$. For extractions, TRIzol reagent (Invitrogen) and DNase I (Sigma-Aldrich) were used according to the manufacturers' instructions.

First-strand cDNA was synthesized using SuperScript II Reverse Transcriptase (Invitrogen) with oligo $(\mathrm{dT})_{15}$ primers according to the manufacturer's instructions. Amounts of cDNA were determined by RT-qPCR using SYBR Green I in a MiniOpticon real-time PCR detection system (Bio-Rad).

Gdh and $A d h$ housekeeping genes, characterized by relatively low expression comparable to esg expression, were used as reference genes to normalize for differences in total cDNA between the samples. The forward and reverse primer sequences used were: Esg-rt1 5'-CGAGTTCTACAGGACCAT CAATCAGC-3' and Esg-rt2 5'-CGCCGATTGGTCTATGGA TGAT-3'; Gdh: Gdh1 5'-TATGCCACCGAGCACCAGATTC C-3' and Gdh2 5'-GGATGCCCTTCACCTTCTGCTTCTT-3'; Adh: Adhd3: 5'-CGGCATCTAAGAAGTGATACTCCCAAAA3' and Adhr3: 5'-TGAGTGTGCATCGAATCAGCCTTATT-3'.

CFX Manager 3.1 software (Bio-Rad, 2012) was used to evaluate the relative gene expression. Inter-run calibrations were used for each panel of experiments since the experiments were conducted for several years and two different models of Bio-Rad qPCR detection systems were used. Two to three independent RNA extractions (biological repeats) per genotype per age (developmental stage) were made. From three to six technical repeats were made for each RNA extraction, including, in some cases, independent c-DNA samples. The averaged results of technical repeats were used for further analysis.

\section{Statistical Analyses}

To compare control and mutant genotypes, Student's $t$-test and the nonparametric, distribution-free Kruskal-Wallis test were used for the analyses of locomotion and the amount of esg transcript. These two tests gave consistent results, so only the results of the Kruskal-Wallis test are reported here. Standard descriptive statistical analysis of life span (Wilmoth and Horiuchi, 1999; Carey, 2003) was performed to determine the mean life span and its accompanying variances, standard deviations and standard errors; the median, minimum and maximum life spans; and the life spans of the lower and upper quartiles, 10 and 90 percentiles (Supplementary Table). Survival 
curves were estimated using the Kaplan-Meier procedure. The nonparametric, distribution-free Mann-Whitney test and Kolmogorov-Smirnov test were used to evaluate the statistical significance of the difference between the survival curves. The Tukey test was used for multiple comparisons when appropriate.

\section{RESULTS}

\section{esg ${ }^{B G 01042}$ Increased Male and Female Life Spans and Locomotion}

A Drosophila melanogaster line with the esg mutation, w[1118]; $P\{G T 1\}$ esg $g^{B G 01042}$ (esgP) and a control line (Control M) with the genotype $w^{1118}$ were used in this study to describe the impact of the gene in the control of life span and aging. The life spans of esgP and Control $\mathrm{M}$ flies were assessed several times. In the two screening experiments performed in the Trudy Mackay laboratory and described in Magwire et al. (2010), the life spans of esgP males were significantly longer than those of the controls, whereas the effect in females was not significant. This result was confirmed in the first experiment and the increase in length of the esgP male life span was further confirmed in the second experiment, performed in our laboratory (Magwire et al., 2010). The results of the two later experiments were presented in Magwire et al. (2010) only as a diagram showing the mean life spans. Here, we present these data in more detail for comparison with the other results; the distributive statistics are given in Table 1 and Supplementary Table (exp. \#1 and \#2) and the survival curves are shown in Figure 1.

Sex-specific and sex-antagonistic effects on life span are not rare (Tricoire et al., 2009; Ruiz et al., 2011; Roshina et al., 2014; Schriner et al., 2014; Shaposhnikov et al., 2015); for example, of 59 mutations increasing life span, $70.7 \%$ affected males and females differently (Magwire et al., 2010). In the initial screens and in our first experiment (Magwire et al., 2010), the effects of $e g^{B G 01042}$ on the life span of female flies were not statistically significant leading to the conclusion that esg ${ }^{B G 01042}$ affects life span in a male-specific manner. However, the sample sizes in these experiments were small, and weak effects could have gone unnoticed. To better understand whether the effects of esg $^{B G 01042}$ on life span are strictly male-specific, we re-measured life span in esgP and Control $\mathrm{M}$ males and females (Table 1 and Supplementary Table, exp. \#3; Figures 2A,D). A significant increase in life span was detected in mutant males compared with control males as well as in mutant females compared with control females. Nevertheless, the positive effect of the mutation was considerably smaller in females $(33 \%$ of the control mean life span) than in males (58\%). This result was confirmed in two other experiments (Table 1 and Supplementary Table, exp. $\# 4$ and \#5; Figures 2B,C,E,F). Overall, the positive effect of the mutation varied from 22 to $96 \%$ in males and from 4 to $33 \%$ in females, the average effects being 55 and $17 \%$, respectively. Comparison of survival curves in all experiments indicated that the mutation slowed aging in both males and females (Figure 2). The maximum life span of mutant males was $40 \%$ higher and the maximum life span of mutant females was $17 \%$ higher than the controls (Table 1).
The life span of heterozygous esgP/Control $\mathrm{M}$ males and females was significantly higher compared to homozygous Control $\mathrm{M}$ flies and significantly lower compared to esgP flies (Table 1 and Supplementary Table, exp. \#4; Figures 2B,E), which indicated a co-dominance of the esg ${ }^{B G 01042}$ mutation.

To further assess the effects of esg $g^{B G 01042}$ on life span, we compared the life spans of mated esgP and Control $\mathrm{M}$ flies (Table 1 and Supplementary Table, exp \#6, exp. \#7). While, technically, experiments with unmated flies are easier, mated flies represent a better model of the naturally occurring way of life. A significantly increased life span was detected in mated mutant males compared with control males (Figure 3A). To verify this result, the experiment was repeated and the life span of mutant males was again significantly higher than that of control males (Figure 3B). The life span of mutant mated females was not different from the life span of control females (Figure 3C). To verify this result, the experiment was repeated and identical results were obtained (Figure 3D). In mated males, the average positive effect of the mutation was somewhat lower (43\%) than that in unmated males. A comparison of the survival curves in experiments with mated males indicated that the mutation slowed aging (Figures 3A,B). In mutant males, the maximum life span was $40 \%$ higher, on average, than that of the controls (Table 1).

General locomotor activity decreases with normal aging in animals and is often considered a marker of age and health (Ridgel and Ritzmann, 2005). In unmated males and females and in mated males, survival curves indicated that the mutation slowed down aging. We assessed the effect of $\mathrm{esg}^{\text {BG01042 }}$ on locomotor activity in unmated males and females and mated males to double check if the rate of aging is actually affected in these cases. After the 20th day of life, an age-dependent decline in locomotor activity was observed in both the mutant and control flies, regardless of their mating status, in good agreement with our prediction (Figure 4). However, in mutant flies with an increased life span, this age-dependent decline was followed by an elevation in locomotion recorded in the 50-day-old individuals compared with the controls (Figure 4). Locomotion was significantly increased in all 50-day-old mutant flies with an increased life span, compared with the controls. These results indicate that, in old mutant flies, aging was effectively slowed down or reversed. In addition, in mutant males, in which the mutation affected life span most strongly, locomotion increased starting at 20 days (Figure 4). Overall, the effects of locomotion paralleled those of life span, and a higher level of mobility in older mutant flies confirmed our hypothesis, which was based on an initial comparison of survival curves, that $e s g^{B G 01042}$ would slow aging.

\section{esg ${ }^{B G 01042}$ Decreases esg Transcript Amounts}

To understand the molecular basis of differences in life span caused by the mutation, we assessed the effect of esg $g^{B G 01042}$ on esg transcript amounts (Figure 5). Only one protein coding esg transcript and one polypeptide have been reported (http:// flybase.org/reports/FBgn0001981.html). Transcript amounts of 
TABLE 1 | Distributive statistics of life span.

\begin{tabular}{lll}
\hline Exp. no Line $\quad$ Mean Standard error Median Percentile 90 & $\begin{array}{c}P \text {-values for comparisons } \\
\text { with the corresponding control lines }\end{array}$ \\
\cline { 3 - 3 } & $\begin{array}{c}\text { Mann-Witney } \\
\text { test }\end{array}$ & $\begin{array}{c}\text { Kolmogorov-Smirnov } \\
\text { test }\end{array}$
\end{tabular}

\section{esg MUTATION, UNMATED MALES}

\begin{tabular}{|c|c|c|c|c|c|c|c|c|c|}
\hline \multirow[t]{4}{*}{1} & Control & 50 & 50 & 1.9 & 54 & 61 & & & A \\
\hline & esgP & 50 & 61 & 2.1 & 63 & 77 & $<0.0001$ & $<0.001$ & B \\
\hline & rev3 & 49 & 57 & 2.8 & 52 & 77 & 0.7029 & $<0.005$ & A \\
\hline & rev5 & 49 & 65 & 1.8 & 66 & 78 & $<0.0001$ & $<0.001$ & $\mathrm{~B}$ \\
\hline \multirow[t]{3}{*}{2} & Control & 150 & 46 & 1.2 & 48 & 63 & & & A \\
\hline & esgP & 150 & 70 & 1.6 & 79 & 86 & $<0.0001$ & $<0.001$ & $\mathrm{~B}$ \\
\hline & rev3 & 150 & 44 & 0.7 & 44 & 51 & 0.0840 & $<0.005$ & A \\
\hline \multirow[t]{2}{*}{3} & Control M & 100 & 36 & 1.4 & 38 & 53 & & & \\
\hline & esgP & 100 & 57 & 1.9 & 66 & 72 & 0.0001 & $<0.001$ & \\
\hline \multirow[t]{3}{*}{4} & Control M & 150 & 28 & 1.1 & 26 & 48 & & & A \\
\hline & esgP & 150 & 55 & 1.3 & 58 & 71 & 0.0001 & $<0.001$ & $\mathrm{~B}$ \\
\hline & Control $\mathrm{M} \times \mathrm{esg} \mathrm{P}$ & 50 & 45 & 2.6 & 52 & 63 & 0.0006 & $<0.005$ & $\mathrm{C}$ \\
\hline \multirow[t]{4}{*}{5} & Control M & 195 & 45 & 1.2 & 48 & 61 & & & A \\
\hline & esgP & 110 & 66 & 1.5 & 69 & 83 & 0.0001 & $<0.001$ & $\mathrm{~B}$ \\
\hline & Rev3 & 150 & 49 & 1.4 & 48 & 76 & 0.1856 & $<0.1$ & A \\
\hline & Rev5 & 205 & 56 & 1.2 & 59 & 77 & 0.0001 & $<0.001$ & $\mathrm{C}$ \\
\hline \multicolumn{10}{|c|}{ esg MUTATION, UNMATED FEMALES } \\
\hline \multirow[t]{2}{*}{1} & Control M & 50 & 62 & 2.1 & 67 & 77 & & & \\
\hline & esgP & 50 & 65 & 1.8 & 67 & 83 & 0.5533 & $>0.10$ & \\
\hline \multirow[t]{2}{*}{3} & Control M & 100 & 36 & 1.7 & 38 & 53 & & & \\
\hline & esgP & 100 & 48 & 1.6 & 55 & 65 & 0.0001 & $<0.001$ & \\
\hline \multirow[t]{3}{*}{4} & Control M & 150 & 41 & 1.2 & 45 & 59 & & & A \\
\hline & esgP & 150 & 51 & 1.5 & 56 & 72 & 0.0001 & $<0.001$ & B \\
\hline & Control $\mathrm{M} \times \mathrm{esgP}$ & 50 & 49 & 2.2 & 54 & 63 & 0.0003 & $<0.001$ & $\mathrm{C}$ \\
\hline \multirow[t]{4}{*}{5} & Control M & 114 & 45 & 1.5 & 47 & 66 & & & A \\
\hline & esgP & 124 & 58 & 1.6 & 61 & 77 & 0.0001 & $<0.001$ & $\mathrm{~B}$ \\
\hline & Rev3 & 114 & 50 & 1.9 & 51 & 76 & $\underline{0.0243}$ & $<0.1$ & A \\
\hline & Rev5 & 115 & 56 & 1.3 & 54 & 72 & 0.0001 & $<0.001$ & B \\
\hline \multicolumn{10}{|c|}{ esg MUTATION, MATED MALES } \\
\hline \multirow[t]{2}{*}{6} & Control M & 60 & 30 & 1.9 & 30 & 50 & & & \\
\hline & esgP & 60 & 46 & 2.2 & 50 & 66 & 0.0001 & $<0.001$ & \\
\hline \multirow[t]{2}{*}{7} & Control M & 60 & 33 & 1.5 & 36 & 45 & & & \\
\hline & esgP & 60 & 44 & 2.3 & 46 & 67 & 0.0001 & $<0.001$ & \\
\hline \multicolumn{10}{|c|}{ esg MUTATION, MATED FEMALES } \\
\hline \multirow[t]{2}{*}{6} & Control M & 60 & 37 & 1.6 & 38 & 50 & & & \\
\hline & esgP & 60 & 37 & 1.9 & 37 & 57 & 0.9581 & $>0.1$ & \\
\hline \multirow[t]{2}{*}{7} & Control M & 60 & 34 & 1.4 & 37 & 45 & & & \\
\hline & esgP & 60 & 34 & 1.9 & 34 & 50 & 0.8011 & $>0.1$ & \\
\hline \multicolumn{10}{|c|}{ esg RNA-i KNOCKDOWN IN THE NERVOUS SYSTEM, UNMATED MALES } \\
\hline \multirow[t]{3}{*}{8} & Control K2 & 100 & 51 & 1.6 & 52 & 70 & & & \\
\hline & Kdw2 & 100 & 47 & 0.9 & 46 & 59 & 0.0112 & $<0.005$ & \\
\hline & Kdw3 & 100 & 58 & 1.8 & 58 & 79 & 0.0021 & $<0.025$ & \\
\hline \multirow[t]{2}{*}{9} & Control 2 & 100 & 61 & 1.5 & 64 & 74 & & & \\
\hline & Kdw3 & 100 & 65 & 1.7 & 67 & 85 & 0.0351 & $<0.05$ & \\
\hline & A-i KNOCKDOWN & IN TH & NEF & SYS & JMAT & MALE & & & \\
\hline 8 & Control K2 & 100 & 76 & 2.0 & 82 & 97 & & & \\
\hline & Kdw2 & 100 & 71 & 2.1 & 77 & 92 & 0.0111 & $<0.1$ & \\
\hline & Kdw3 & 100 & 84 & 1.6 & 90 & 98 & 0.0004 & $<0.001$ & \\
\hline 9 & Control K2 & 100 & 88 & 1.4 & 92 & 101 & & & \\
\hline & Kdw3 & 100 & 93 & 1.3 & 95 & 105 & 0.0049 & $<0.025$ & \\
\hline
\end{tabular}

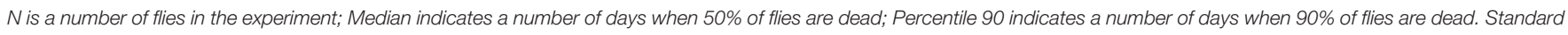

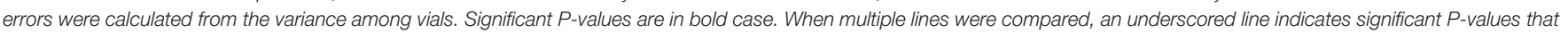

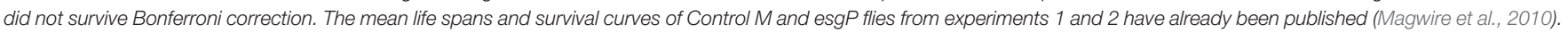

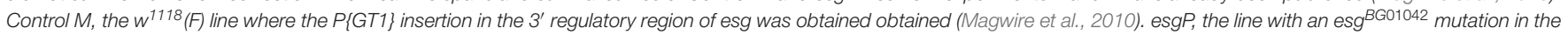

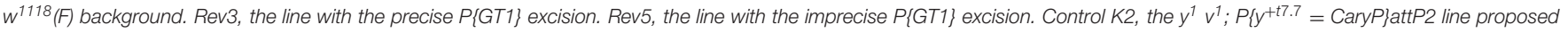

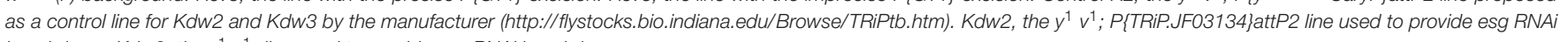
knockdown. Kdw3, the $y^{1} v^{1}$; line used to provide esg RNAi knockdown. 


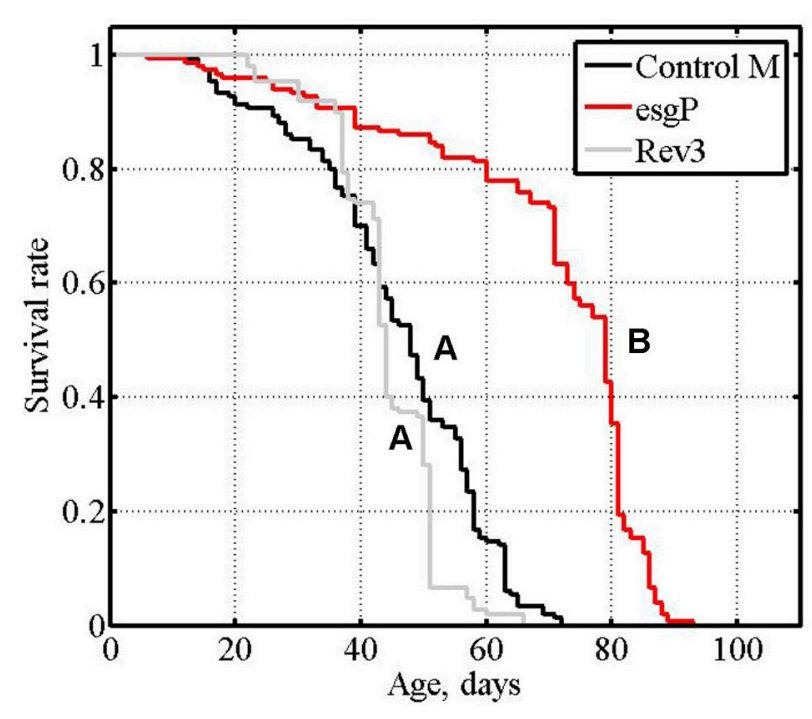

FIGURE 1 | Initial analysis of mutant and revertant lines: survival of unmated males, cumulative data of experiments \#1 and \#2. Letters A and B indicate the results of Tukey tests for significant differences between control, mutant and revertant lines. Lines with the same letter are not significantly different from each other.

esg in adult flies are very low and are slightly higher in males than in females due to transcription in testes (http://flybase. org/reports/FBgn0001981.html). The effects of esg ${ }^{B G 01042}$ on life span were the strongest in unmated males. For these reasons, we concentrated our main efforts on measuring transcription rates in unmated males. In our initial experiment conducted simultaneously with experiment \#3 measuring life span, we compared total esg transcript amounts in 1-, 10- and 20day-old unmated males with and without the mutation. A significant decrease in esg transcript amounts was found in 10-day-old mutant males compared with controls (Figure 5A). In 1-day-old mutant males, the increase in expression was marginally significant and in 20-day-old males the increase was not statistically significant. Our overall interpretation of these results was that esg ${ }^{B G 01042}$ reduced esg transcript amounts at all ages but, in some cases, quantities of mRNA were too small to reach the level of statistical significance. The statistically significant differences were confirmed when comparing esg transcript amounts in 1-day-old males in the experiment that was conducted a year later, at the same time with experiment \#4 measuring life span (Figure 5B).

We made an attempt to measure transcription rates in 1day-old control and mutant unmated females. Several technical replicates made with the same biological samples demonstrated that the amount of esg RNA is slightly smaller in mutants $(0.9 \pm$ 0.02 vs. $1 \pm 0.02$ relative arbitrary units). Given small absolute amounts of esg mRNA in females and a small difference observed in this single experiment, we did not pursue this line of study any more. Satisfactory enough, the result of the experiment with females was in line with our conclusion that the esg transcription rate is lower in mutants than in controls.
Expression of esg is predominant in embryos (http://flybase. org/reports/FBgn0001981.html). The expression of esg mRNA is persistent throughout embryogenesis, with the highest amounts observed from 2 up to $20 \mathrm{~h}$ of development. Simultaneously with experiment \#5, we evaluated esg transcript amounts in 14-20-hold mutant and control embryos. This moderately large interval allowed us to obtain the overall characteristics of control and mutant embryos and to offset the possible uneven contribution of embryos at different stages of development. The esg transcript amounts were significantly lower in mutant embryos than in control embryos (Figure 5C).

\section{Precise and Imprecise Reversions of esg $^{B G 01042}$ Have Different Effects on Life Span and esg Expression}

Standard substitution crosses with balancers and delta 2-3 source of P element transposase (Robertson et al., 1988) were used to obtain lines with reversions of the esg $g^{B G 01042}$ mutation, while maintaining the co-isogenic background. These crosses were described in more detail in Magwire et al. (2010), the detailed description of revertant lines is given here. Seven lines with reversions of the marker $w^{+}$phenotype were obtained from five males with active transposase. For each line, PCR with primers flanking the site of the initial $P\{G T 1\}$ insertion were used to assess the nature of the reversions. PCR fragment sizes were identical in the control line and in two lines with reversions, indicating precise excisions of the $P\{G T 1\}$ construct. In five other lines with reversions, the PCR fragment sizes were slightly larger, indicating that imprecise excisions occurred (Figure 6). This was further confirmed by sequencing the PCR fragments: in one line (Rev3), the sequence was identical to the standard gene sequence (http://flybase.org/reports/FBgn0001981.html), in the other line with a precise excision (Rev4), three nucleotide substitutions were detected; in lines Rev1.1, Rev1.2, Rev2.1, Rev2.2, and Rev5, insertions of 30-162 bp in length were detected between the duplicated insertion sites. Two revertant lines, Rev3 with the complete restoration of the original genome structure and Rev5 with the $32 \mathrm{bp}$ insertion $602 \mathrm{bp}$ downstream of esg, were kept in stock and used in this study.

The life span of Rev3 males was measured in experiments $\# 1$ and \#2 to demonstrate that the reversion of the mutation was accompanied by the reversion of the mutant phenotype. The life span of Rev3 males was the same as that of Control M males, and the results of Tukey tests for significant differences between the control, mutant and revertant lines proved the causal relationship between the mutation and life span changes (Magwire et al., 2010). Experiment \#5 confirmed the effect of the precise reversion on the male life span and attested that the same effect was observed in females (Table 1, Figures 2C,F).

The life span of Rev5 males was measured in experiments $\# 1$ and \#5 and the life span of Rev5 females was measured in experiment \#5 to compare the effects of precise and imprecise reversions. In experiment \#1, the life span of Rev5 males was not different from that of esgP males $(P=0.1564$ for MannWhitney test). The Tukey test allowed us to divide the lines into two groups: Control M and Rev3 vs. esgP and Rev5 (Table 1). 

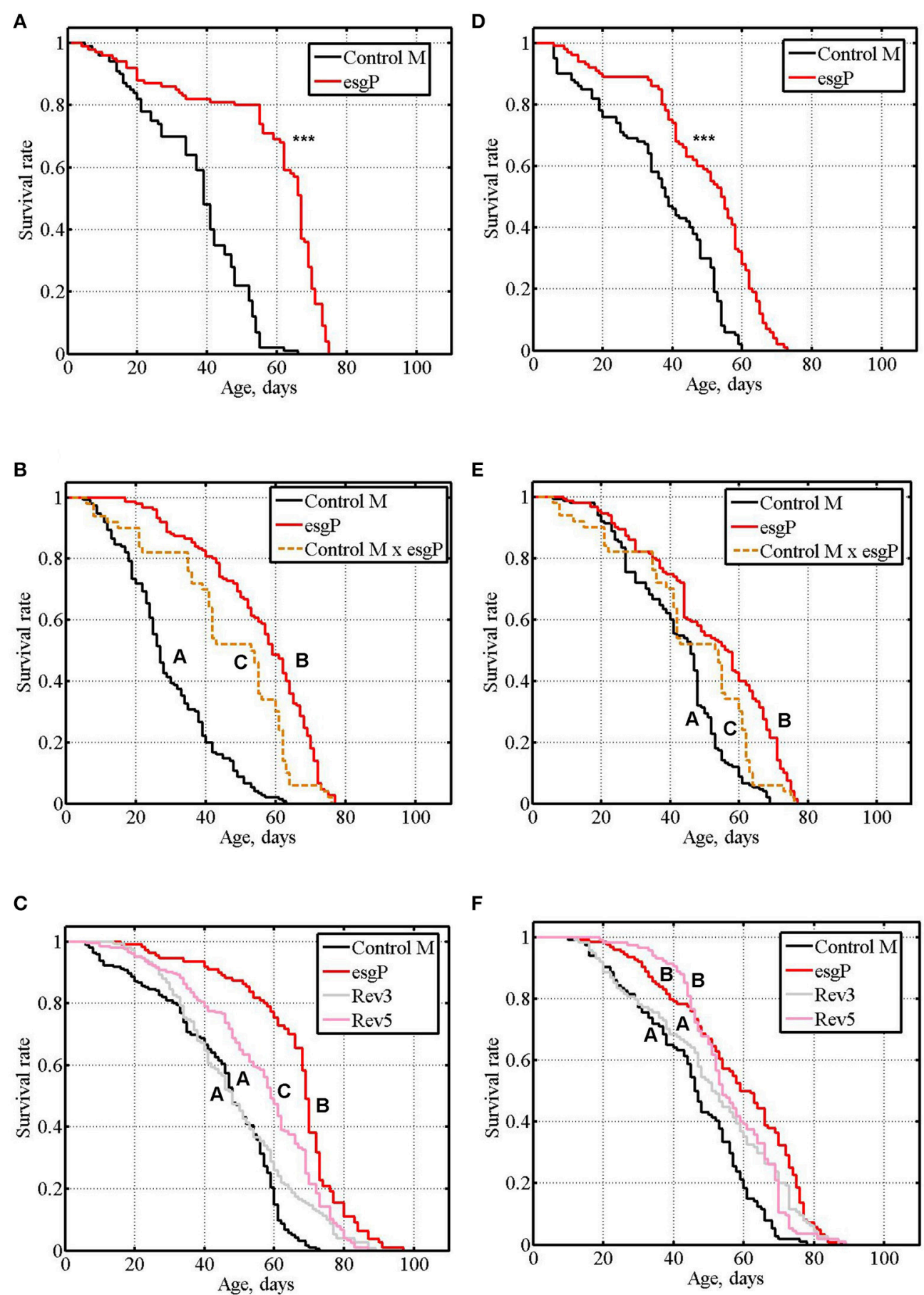

FIGURE 2 | Survival of unmated mutant and revertant flies. (A-C): experiments \#3, \#4, \#5 with males. (D-F): experiments \#3, \#4, \#5 with females. Asterisks denote significant differences with the Control $\mathrm{M}$ line, as determined by the Mann-Whitney test ( $\left.{ }^{\star \star \star} P<0.001\right)$. Letters $\mathrm{A}$, B, and $\mathrm{C}$ indicate the results of Tukey tests for significant differences between different genotypes. Genotypes with the same letter are not significantly different from each other.

In experiment \#5, the life span of Rev5 males was different from both esgP males $(P<0.0001$ for the Mann-Witney test $)$ and control M males (Table 1). The esgP and Rev5 lines divided into different groups, while the Control $\mathrm{M}$ and Rev3 lines remained in the same group (Table 1, Figure 2C). The life span of Rev5 females was not different from the life span of esgP females ( $P=0.1075$ for the Mann-Witney test), and the Tukey test allowed us to divide the lines into two groups: Control $\mathrm{M}$ 


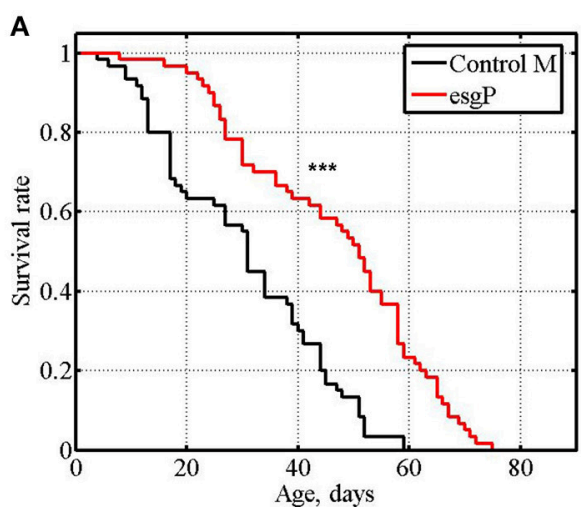

B

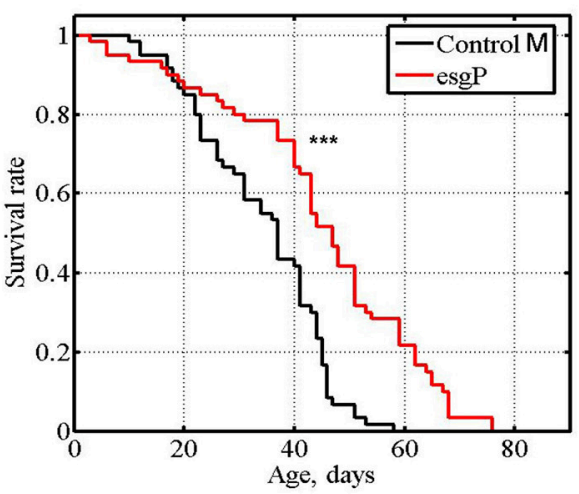

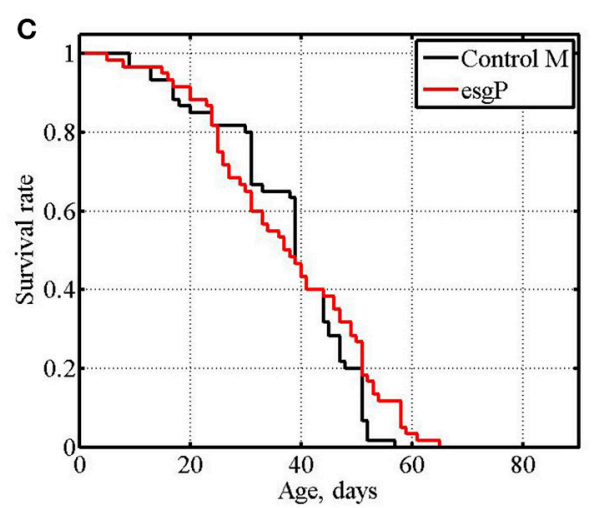

D

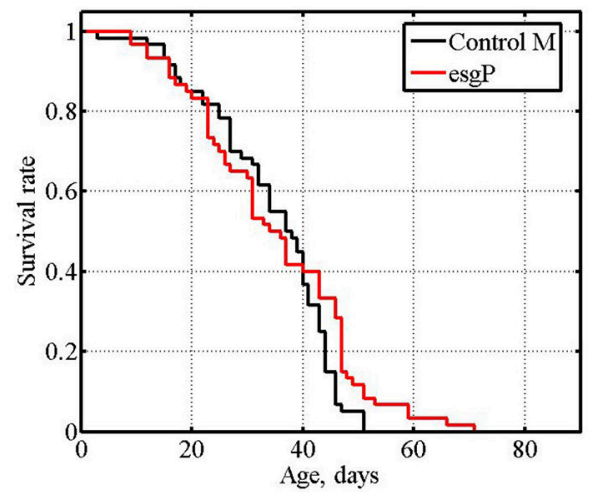

FIGURE 3 | Survival of mated mutant flies. (A,B): experiments \#6, \#7 with males. (C, the Control $\mathrm{M}$ line as determined by the Mann-Whitney test $\left({ }^{* \star *} P<0.001\right)$.

and Rev3 vs. esgP and Rev5 (Table 1, Figure 2F). The results indicated that a small $32 \mathrm{bp}$ insertion located $602 \mathrm{bp}$ downstream of esg had virtually the same effect on male and female life spans as a large 8500 bp $P\{G T 1\}$ insertion.

This conclusion raised the important question of whether the differences and similarities in life spans in control, mutant and revertant lines were paralleled by the differences and similarities in esg transcription levels. We evaluated esg transcript amounts in mutant, control, and revertant 1-day-old males and in 14to 20-h-old embryos (Figures 5B,C). In neither case was the difference significant between the Control $M$ and Rev3 lines or between the esgP and Rev5 lines. The difference between the Control M and Rev5 was marginally significant in males and significant in embryos; the difference between esgP and Rev3 was marginally significant in males. Visually, Control $\mathrm{M}$ and Rev3 formed one group and esgP and Rev5 formed a second group, however, the Tukey test did not confirm this. Despite some statistical failures, these results indicate that the precise reversion of the mutation was accompanied by the reversion of the transcription level, while the imprecise reversion did not affect the transcription level characteristic for the mutation. This gives us serious reasons to say that a decrease in esg transcription underlies the decrease in the life span of mutants. Additionally, these results confirmed that a small $32 \mathrm{bp}$ insertion and a large $8.5 \mathrm{~kb} P\{G T 1\}$ insertion located
602 bp downstream of esg affects esg expression in the same way.

\section{esg Knockdown in the Nervous System Increases Male and Female Life Span}

Analysis of the esg mutation and its precise reversion demonstrated that the decrease in esg transcription was causally associated with the increase in life span. Transcript levels were measured, either in the whole bodies of adult flies or in whole embryos, and what changes in which tissues were responsible for the observed effects remains unknown. Given that esg mRNA is not abundant and, hence, precise evaluation of its quantity in individual tissues of mutant and control flies is rather difficult technically, we used the binary GAL4-UAS system to induce RNAi knockdown of esg and in this way to decrease its expression in a tissue-specific manner. To get a broader understanding of the knockdown effects, we used three independent lines with RNA interference in the hope that at least one of them would provide a decrease in esg transcription similar to the effect of the mutation. We were primarily interested in understanding the role of neuronal genes and the nervous system in life span control. Based on this, we decided to evaluate the effects of the esg knockdown in the nervous system on male and female life spans. Life span of unmated males and females was selected as a tester 

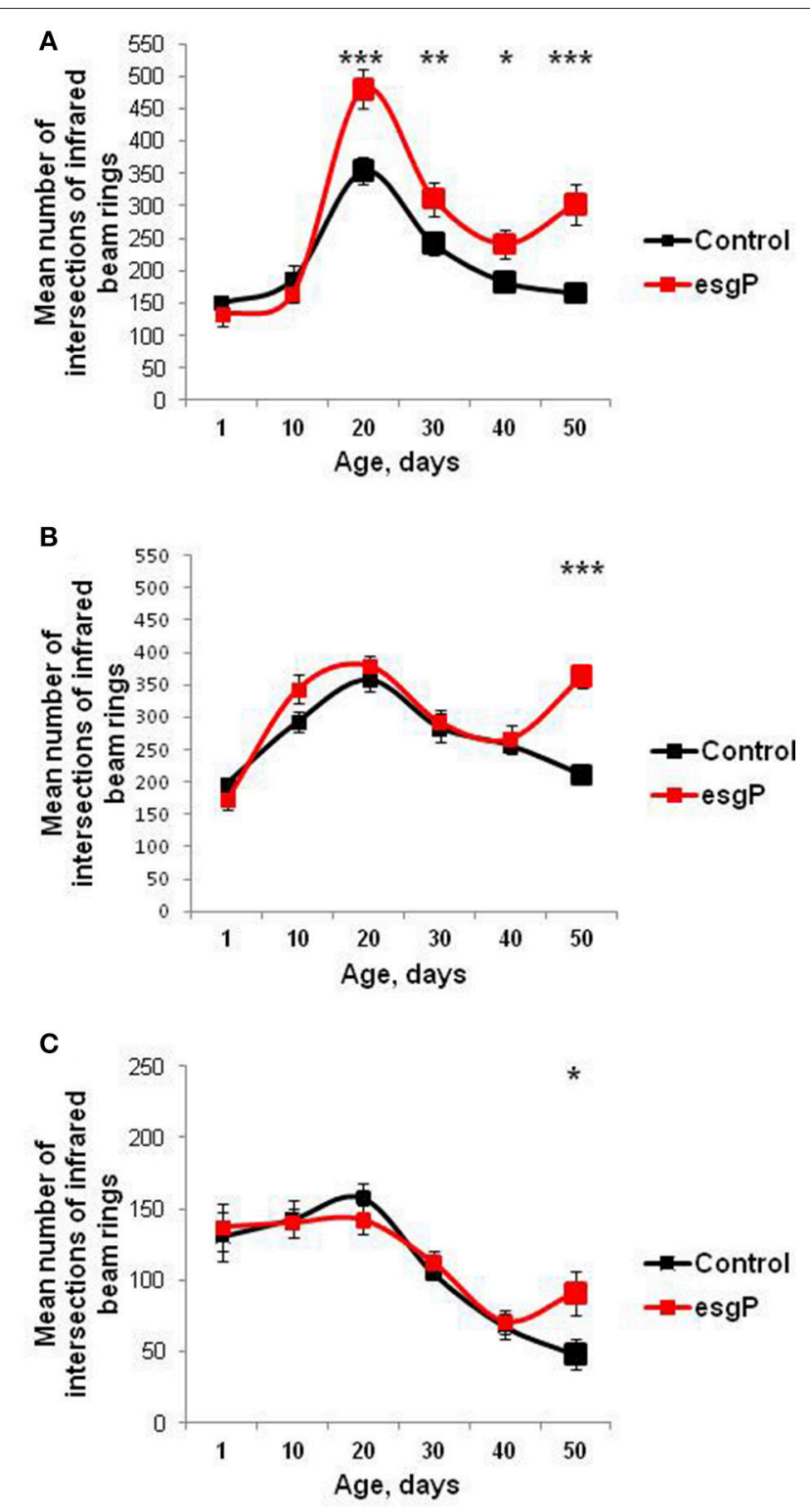

FIGURE 4 | Age-dependent locomotion of mutant flies. (A) Locomotion of unmated males (B) Locomotion of unmated females. (C) Locomotion of mated males. Asterisks denote significant differences compared with the Control $\mathrm{M}$ line, as determined by the Kruskall-Wallis test $\left({ }^{\star} P<0.05 ;{ }^{* \star} P<\right.$ $\left.0.01 ;{ }^{\star \star \star} P<0.001\right)$.

trait in these experiments because, technically, experiments with unmated flies are easier and the effects generally tend to be more obvious. Also, both unmated males and females demonstrated response to changes in the esg function, whereas among mated flies, only males were affected.

$w^{1118} ; P\{G D 1437\} v 9793, y^{1} v^{1} ;$ P\{TRiP.JF03134\}attP2, $y^{1} v^{1}$; and P\{TRiP.HMS00025\}attP2 lines with transgenes encoding esg hairpins, and the corresponding control lines suggested by the manufacturers were used to evaluate the effects of the knockdown. A well-known line, well-known line, $P\left\{w^{+m W . h s}=G a w B\right\} e l a v{ }^{C 155} w^{1118} ; P\left\{w^{+m C}=U A S-D c r-2 . D\right\} 2$,
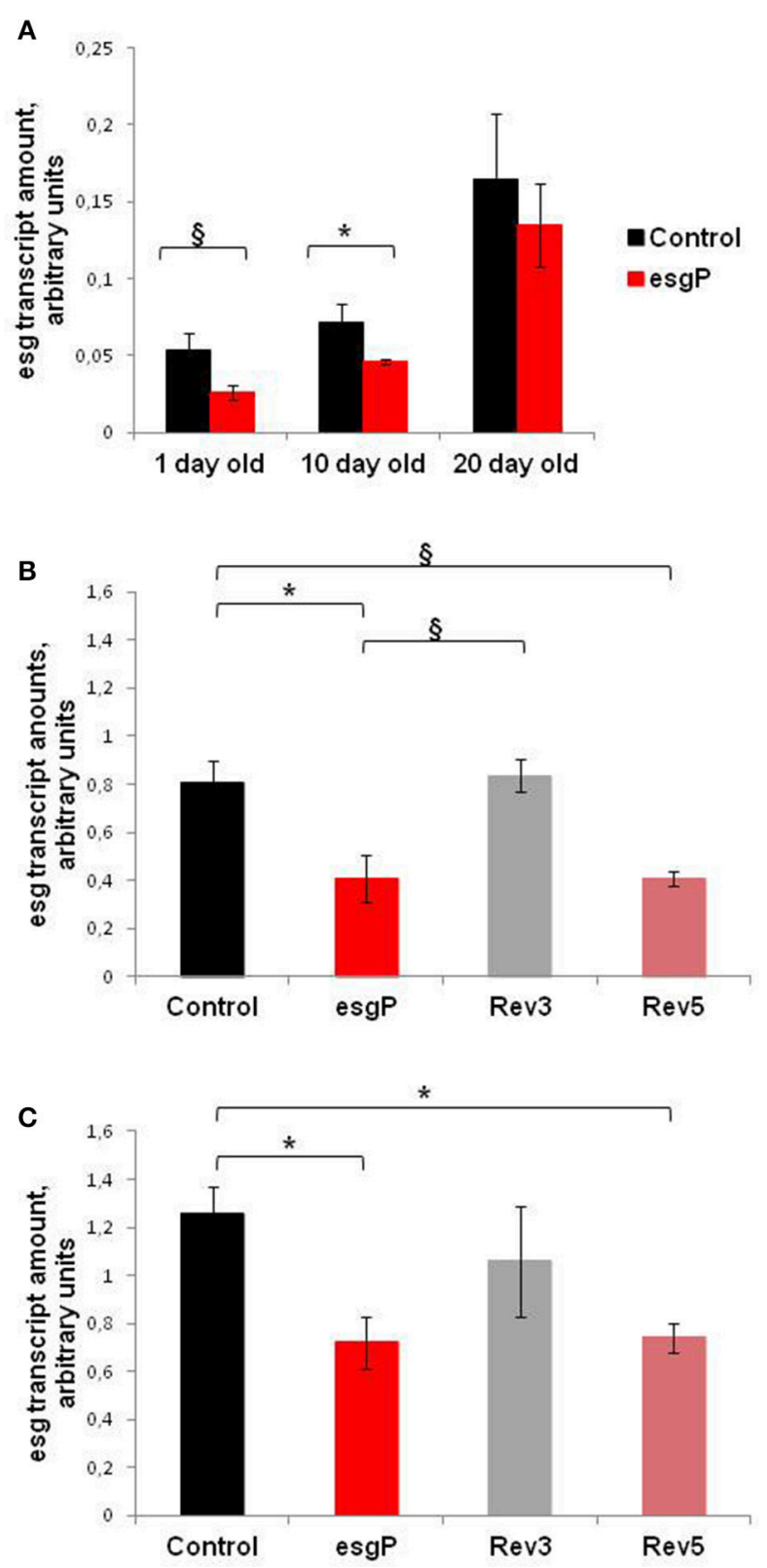

FIGURE 5 | esg transcript amounts in unmated mutant and revertant flies and embryos. Transcript amounts in (A) unmated males of different ages; (B) 1 day-old unmated males; (C) embryos. Two biological repeats were done in the first experiment with 1- and 20- day-old males (A). Three biological repeats were done in the first experiment with 10-day-old males (A), in the second experiment with 1-day-old males (B), and in the experiment with embryos (C). The paragraph sign denotes marginally significant differences $\left({ }^{\S} P<0.10\right)$ and the asterisk denotes significant differences $\left({ }^{\star} P<0.05\right)$ as determined by the Kruskall-Wallis test.

was used to induce the expression of transgenic constructs in all the neurons. The presence of an additional, inducible copy of Dicer in its genome provided better interference. Hybrid progeny used for life span measurements were denoted as Kdw1, Kdw2, Kdw3, Control K1, and Control K2. 


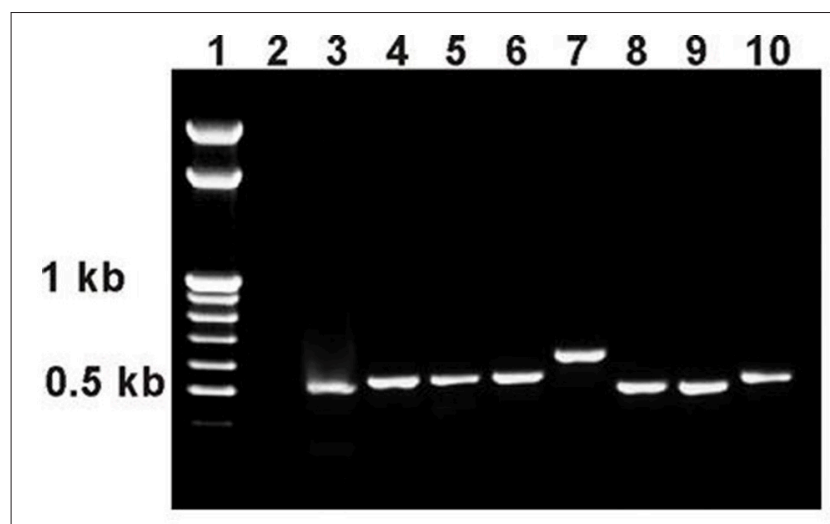

FIGURE 6 | PCR analysis of esg structure in revertant lines. PCR primers esg1 and esg2 were used with an expected product size of $508 \mathrm{bp}$. 1: marker (GeneRuler ${ }^{\mathrm{TM}} 100$ bp Mass DNA Ladder, Fermentas); 2: esgP (negative control, an expected PCR product of approximately $9 \mathrm{~kb}$ is not produced under the PCR conditions used); 3: Control M; 4: Rev1.1; 5: Rev1.2; 6: Rev2.1; 7: Rev2.2; 8: Rev3; 9: Rev4; 10: Rev5.

In experiment $\# 8$, different results were obtained with the three lines providing esg knockdown. In the first case (Kdw1), esg knockdown in the nervous system caused lethality. In the second case (Kdw2), in both males and females, life span was significantly lower compared with the controls (Table 1 and Supplementary Table, Figures 7A,C). In the third case (Kdw3), in both males and females, life span was significantly higher compared with the controls (Table 1 and Supplementary Table, Figures $7 \mathbf{A}, \mathbf{C})$. In the two later cases differences were rather small. The positive effect was reproduced in experiment \#9 (Table 1 and Supplementary Table, Figures 7B,D).

Our results demonstrated that reduced esg expression in the nervous system may cause both deleterious and beneficial effects. It would be logical to suggest that the strength and the direction of the effect depend on the effectiveness of the knockdown provided by different lines. Kdw1 might provide the strongest knockdown and the lowest rate of esg transcription incompatible with survival, Kdw2 might provide an intermediate level of knockdown, and Kdw3 might provide the slightest level of knockdown, resulting in a beneficial finetuning of the level of esg transcription. However, according to the manufacturer's description, $\mathrm{Kdw} 3$ is supposed to provide a stronger knockdown than Kdw2 (http://flystocks.bio. indiana.edu/Browse/RNAi/RNAi_D_M.php). A rather specific suggestion that an intermediate level of esg transcription in the nervous system is the most beneficial for longevity might account for the results we obtained. A similar hypothesis explained our data on the effects of Lim 3 expression on life span (Rybina and Pasyukova, 2010; Rybina et al., 2017). Unfortunately, the level of esg expression both in the central nervous system (heads) and peripheral nervous system (carcasses) of adult flies is extremely low (http://flybase.org/reports/FBgn0001981.html), which makes the direct evaluation of the knockdown effects in these tissues with a precision necessary for statistically reliable comparisons rather tricky.
Overall, the data obtained in the experiments with esg knockdown corroborated data obtained in the experiments with the esg mutation: a certain level of decline in esg expression had beneficial effects on survival and aging.

\section{DISCUSSION}

\section{esg Affected Drosophila Longevity}

In this study we demonstrated that esg, a gene encoding a Snailtype transcription factor, increased life span and slowed down aging. The esg mutation most strongly affected unmated males, and, to a lesser extent mated males and unmated females. It was not detected at all in mated females. We also demonstrated that the precise reversion of the esg mutation was associated with the restoration of the control phenotype, both in males and in females, and thus confirmed the causal relationships between the mutation and the life span changes reported earlier (Magwire et al., 2010). However, effects of this single mutation, esg $^{B G 01042}$, on life span are not formally sufficient to assert that esg is involved in life span control. Indeed, it was shown that the insertion located far enough downstream from the structural part of the gene is causally associated with life span, but whether this insertion affects the function of this particular gene remained unknown. Microarray analysis failed to reveal changes in esg transcription rate in esg ${ }^{B G 01042}$ mutants, which could be explained by insufficient power of the method (Magwire et al., 2010). We demonstrated that esg ${ }^{B G 01042}$ affected gene transcription rate and proved that the decrease in esg expression is causally related to the life span increase. This is a crucial result which for the first time demonstrated that esg is involved in life span control.

Though there is no doubt that the $P\{G T 1\}$ insertion affected esg expression, there remained a possibility that it also affected expression of another gene located downstream of esg and that it was this change that caused the observed increase in life span. However, there are no genes close to esg, the nearest downstream neighbor is CG15258, located at a distance of $27,055 \mathrm{bp}$. Information about this gene is scarce; it is predominantly expressed in the embryonic central nervous system, embryonic/larval midgut and adult testes (http://flybase. org/reports/FBgn0032563.html). Given the distance between the $P\{G T 1\}$ insertion and CG15258, remote effects of $P\{G T 1\}$ on CG15258 expression seem improbable, though we cannot fully exclude them.

Determining if other esg mutations have effects on fly longevity could be suggested in order to confirm the involvement of the gene in lifespan control. Several insertion mutations affecting esg are publicly available (http://flybase.org/reports/ FBgn0001981.html). However, the answer to this question is already known. In the initial screen of mutations affecting life span (Magwire et al., 2010), seven other insertion mutations in esg affected life span, though in the opposite direction. All seven mutations were in different locations from the mutation associated with increased life span. Mutations decreasing life span are generally assumed to be deleterious and affect various life history traits including fitness, while mutations increasing life span are likely to have specific effects on the trait and, as such, are 

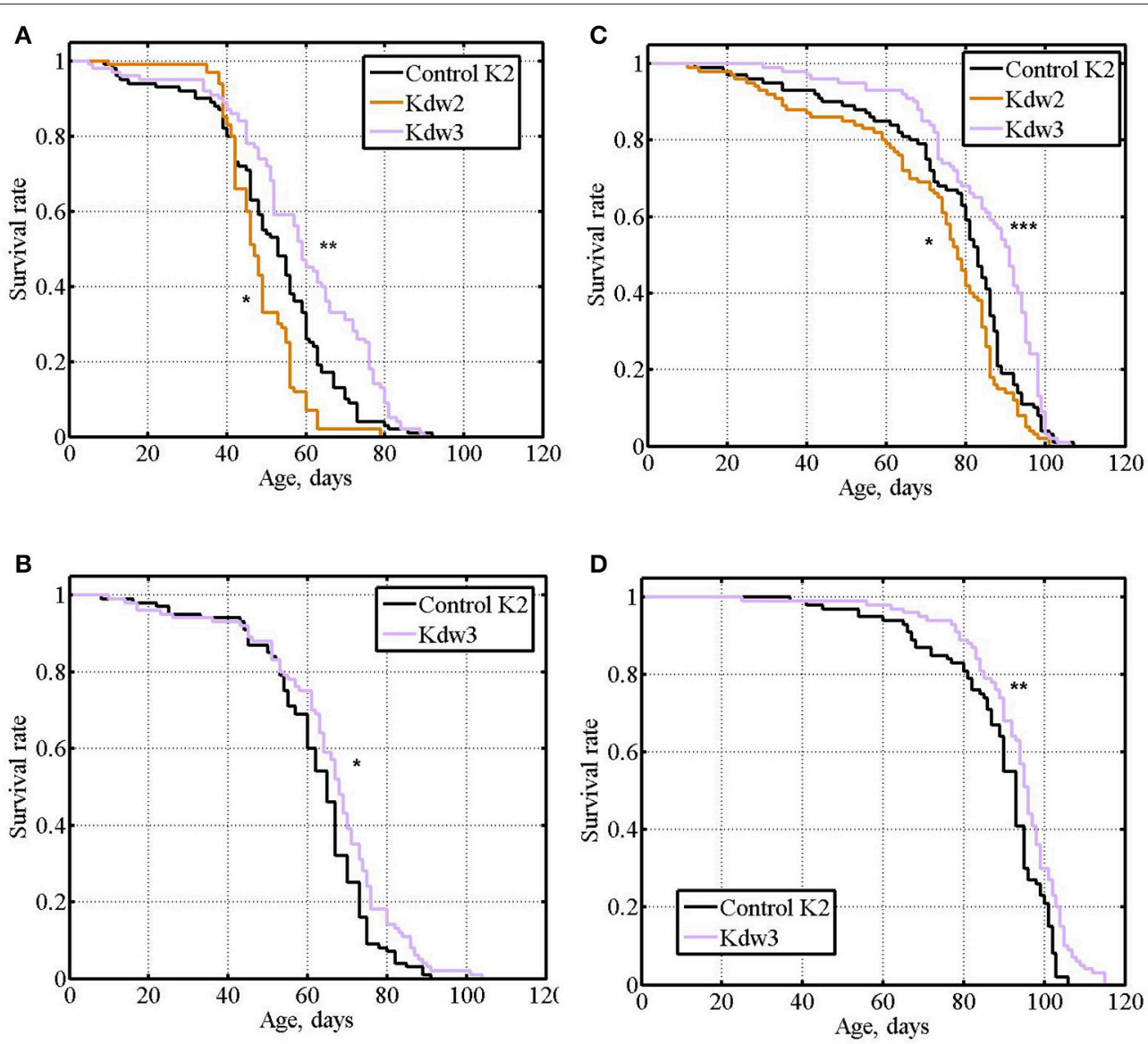

FIGURE 7 | Survival of unmated flies with esg knockdown in the nervous system. (A,B): experiments \#8, \#9 with males. (C,D): experiments \#8, \#9 with females. Asterisks denote significant differences with the Control K2 line as determined by the Mann-Witney test $\left({ }^{\star} P<0.05 ;{ }^{* \star} P<0.01 ;{ }^{\star \star \star} P<0.001\right)$.

more interesting for the study of the genetic control of life span. Still, the fact that eight independent mutations in esg affect life span reinforces the conclusion that this gene is involved in the control of life span. Experiments with gene-specific knockdowns provided final, independent proof of the involvement of esg in life span control.

Of note, the only esg mutation associated with an increased life span was in a slightly different genetic background compared with the mutations associated with decreased life span, which indicate unknown epistatic interactions (Magwire et al., 2010). This evidence agrees with our data showing that the mutations in shuttle craft had different effects on life span in different genetic backgrounds (Pasyukova et al., 2004; Roshina et al., 2014) and the data from other researchers. For example, naturally segregating genes interact epistatically with Sod, a well-known aging gene, to modify its ability to extend longevity (Spencer et al., 2003). The genetic basis of these epistatic interactions remains unknown, but, obviously, esg is expected to interact with other genes because it encodes a transcription factor.

Transcriptional cascades are key regulatory mechanisms (Skeath and Thor, 2003; Jothi et al., 2009). Transcription factors such as FOXO, HSF-1, HIF-1, NFkB, TFEB, and others participate in different signaling cascades and are crucial for the regulation of longevity and aging (for reviews see Alcedo et al., 2013; Bhatt and Ghosh, 2014; Lapierre et al., 2015). Previously, we demonstrated that two genes, shuttle craft and Lim3, which encode transcription factors involved in the development of the nervous system, affect Drosophila life span (Pasyukova et al., 2004; Roshina et al., 2014; Rybina et al., 2017). New data from this study adds one more gene to this list.

Our results demonstrated that a decrease in esg transcription in the nervous system prolonged life span, thus confirming that the neuronal function of esg is indeed relevant to life span control. Microarray analysis revealed approximately $100 \mathrm{esg}$ primary targets whose transcription was induced or repressed by neuronal esg overexpression in Drosophila larvae (HekmatScafe et al., 2005). Targets of esg encoded enzymes involved in the biosynthesis of neurotransmitters, neuropeptides, cationic transporters and other proteins. Among others, genes involved in the defense/immune response were both up- and downregulated. Of the genes known to be involved in life span control, at least two genes associated with increased life span, heat shock protein 26 (hsp26) and NAD-dependent methylenetetrahydrofolate dehydrogenase $(\mathrm{Nmdmc}$ ), increased transcription (Wang et al., 
2004; Yu et al., 2015). It is unclear whether transcription of a similar pool of genes would be affected by a reduction in esg expression or whether the same effects would be observed at other developmental stages and in adults. Obviously, an opposite effect, if any, on $h s p 26$ and Nmdmc transcription would be expected upon esg knockdown, which means that changes in the expression of other esg targets should lead to increased life span in this case, or that esg effects in $h s p 26$ and Nmdmc in embryos and adults would be different compared to in larvae.

In our study, esg knockdown affected the nervous system at all stages of development. It remained undetermined at which stage a decrease in esg transcription was essential for an increase in life span. Normally, esg transcription is low in adult heads and carcasses, which comprise the central and peripheral nervous systems (http://flybase.org/reports/FBgn0001981.html), and its further reduction by knockdown probably reduces it almost to zero. To the best of our knowledge, nothing is known about the vital esg neuronal functions in adult flies, and though it does not mean that these functions do not exist, this fact emphasizes that reducing esg transcription to a virtually negligible level in the adult nervous system is not deleterious. The best known neuronal function of Esg and other Snail family proteins is to control asymmetric neuroblast division during embryonic development (Ashraf and Ip, 2001; Cai et al., 2001, 2003; Wodarz and Huttner, 2003) via two distinct highly conserved mechanisms: one functions throughout mitosis and is implemented via the control of inscuteable and string, the other acts during anaphase/telophase and is inscuteable-independent. There are data indicating that during embryogenesis esg has other functions related to the development of the nervous system (Hartl et al., 2011; Kim et al., 2015; Ramat et al., 2016). One might suggest that it is a decrease in esg transcription at the developmental stages, in particular, at the embryonic stage that matters for longevity. We previously suggested that the long-term, carry-over effects of alterations in embryonic gene expression might be epigenetically inherited in cell lineages or, alternatively, might affect transcriptional cascades that predetermine structural and functional properties of the adult nervous system (Roshina et al., 2014). Our preliminary data showed that insertion mutations in genes that are also involved in the control of asymmetric neuroblast division, such as inscuteable, shaggy, and $a P K C$, are also able to increase life span; however, these results only represent the first steps toward investigating the molecular mechanisms underlying esg effects on life span. Interestingly, ectopic neuronal expression of esg is a general seizure suppressor and esg must be ectopically expressed during nervous system development to produce adults that are less seizure prone (Hekmat-Scafe et al., 2005). Our suggestions agree well with these data.

The decrease in esg transcription in the nervous system caused by RNAi knockdown affected life span to a much lesser degree than the decrease in esg transcription caused by the esg mutation. Evidently, in mutant flies, other tissues were also involved in the implementation of the effects of esg on life span. As a transcription factor, Esg affects many aspects of fly physiology. Effects of the $e s g^{B G 01042}$ mutation on gene transcription were consistent with highly pleiotropic functions of esg (Magwire et al., 2010). However, esg transcription is predominantly limited to just a few tissues and organs: the nervous system (embryos and larvae), gastrointestinal tract (all stages), and testis (adults) (http://www.flymine.org/flymine/report.do?id=1040284\&trail= \%7c1040284). These general characteristics, however, do not shed light on the exact genetic and metabolic pathways underlying the changes in life span. In recent years, significant attention has been drawn to the fact that esg is expressed in stem cells in several tissues, including intestinal stem cells (ISCs), cyst stem cells and male germline stem cells and is a primary component of testis stem cells (Loza-Coll et al., 2014; Voog et al., 2014; Loza-Coll and Jones, 2016). The gastrointestinal tract of multicellular animals was recently recognized as an organ critically important for the control of homeostasis, life span, and aging (Jasper, 2015). In the adult midgut, Esg is expressed in ISCs, and loss of Esg causes their rapid differentiation, whereas an increase in Esg expression locks ISCs into a stem cell state (Korzelius et al., 2014; Loza-Coll et al., 2014). Esg is thus a major repressor specifying whether ISCs remain undifferentiated or commit to differentiation. We hypothesize that a slight decrease in esg expression, such as was observed in esg ${ }^{B G 01042}$ mutants, might shift the ISCs metabolism toward differentiation, thus providing rejuvenation of the gastrointestinal tract and slowing down aging. Given that the effects of $e g^{B G 01042}$ on longevity were more pronounced in males, one might also suggest that similar rejuvenation observed in testes upon decrease in esg expression was responsible for the sex-specificity of aging of esg mutants. We also hypothesize that the accelerated rejuvenation of the germline stem cells in testes induced by esg mutation might have a more pronounced effect on the rate of aging in unmated males because crossing and accompanying expenditure of germ cells partially neutralizes the effect of the mutation in mated males. We thus suggest that the difference in effects of the mutation on life spans of unmated and mated males is rather connected to differences in their physiological and behavioral patterns than to differences in expression levels of esg. Further experiments will shed light on the suggested differential mechanisms of life span control.

\section{The Role of the $3^{\prime}$ Regulatory Region in Modulating esg Transcription}

Molecular mechanisms underlying the relationship between gene expression and phenotype are central to understanding the basics of development, life and aging. In this study, we demonstrated that a decrease in esg transcript levels caused by the insertion of a rather large vector construct 602 bp downstream of esg and 1,240 bp downstream of the end of the single esg exon increased survival and slowed aging. The insertion was located far enough from the structural part of the gene but it remains unclear how it affected transcription or the properties of the transcript that set up its stability. To add to the complexity, our results demonstrated that the size of the insertion (32 bp compared to approximately 8,500 bp) had a negligible effect on esg transcription, which indicated that it was disruption of the regular genomic sequence rather than the properties of the inserted fragment that affected esg expression. According to our analysis using databases of Drosophila regulatory elements (http://www.fruitfly.org/seq_ tools/promoter.html; http://www.ifti.org/cgi-bin/ifti/Tfsitescan. 
pl; http://alggen.lsi.upc.es/cgi-bin/promo_v3/promo/promo.cgi? dirDB=TF_8.3), the presence of a 32 bp insertion did not add any putative regulatory sites (promoters, transcriptional regulators binding sites) to the regular genome sequence downstream of esg.

It is not yet well understood how downstream sequences affect gene transcription (Pance, 2013). Enhancers are able to exert their effects, both upstream and downstream, over long distances (Schaffner, 2015). They function as integrated transcription factor binding platforms (Spitz and Furlong, 2012) and are characterized by special chromatin features (Calo and Wysocka, 2013). Polycomb/Trithorax Response Elements (PRE/TREs) also function over long distances and represent protein-binding platforms that are required to maintain gene transcription and to provide epigenetic inheritance of silent and active chromatin states in cell lineages (Schuettengruber et al., 2007). The latter property was especially interesting in the context of suggested carry-over effects of alterations in esg transcription from embryos to adults.

A possible explanation of the crucial effect of the relatively small insertions on esg transcription rates is that they might perturb the integrity of hypothetical protein-binding platforms. According to the FlyBase analysis (FlyBase, 1992) ${ }^{1}$ of modENCODE data (The modENCODE Consortium et al., 2010) the insertion site co-localizes with a possible Transcription Factor Binding Motifs (TFBS) hotspot. At least one of the possible TFBSs (http://flybase.org/reports/FBsf0000298779.html) refers to the Dorsal transcription factor, which may act as an RNA polymerase II distal enhancer binding factor for esg (Bhaskar and Courey, 2002).

A brief analysis (http://alggen.lsi.upc.es/cgi-bin/promo_v3/ promo/promo.cgi?dirDB=TF_8.3) revealed several putative regulatory sites \pm 250 bp from the insertion site of both $P\{G T 1\}$ and the 32 bp DNA fragment remaining after the imprecise excision of $P\{G T 1\}$. Five proteins involved in control of neurogenesis, synaptic structure and function and three proteins involved in the regulation of sex determination, sexual behavior and fertility might potentially bind DNA sequences located around the insertion. Among others, Doublesex (DSX) deserved our special attention, as it was shown that the regulatory region comprising DSX binding site was able to direct fat body expression of the reporter gene located 8,000 bp upstream (Garabedian et al., 1986), that is, demonstrated properties of enhancer. It is possible to suggest that functionally related sites might form enhancer-like platforms whose integrity was violated by insertions, which led to a decrease in esg transcription.

Ten sites with DNA motifs shown to be important for PRE/TRE function (Ringrose and Paro, 2007) were found in this region, indicating a possibility that Zeste, Pleiohomeotic, GAGA Factor/Pipsqueak and Dorsal Switch Protein 1 might bind DNA in this region. However, the density of the putative binding sites of proteins that are characteristic for PRE/TREs was too low to predict a PRE/TRE presence in the region (Ringrose and Paro, 2007). Of note, Zeste was shown to function as an enhancer for snail (Fuse et al., 1999).

We fully recognize that, even the presence of several binding sites of the same protein as well as the presence of several binding

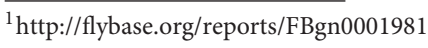

sites of functionally related proteins does not always appear to have functional consequences. Thorough bioinformatic and functional analyses of the region are needed to shed light on the regulatory properties of the DNA sequences downstream of esg. Some results on this point have been already reported. According to the REDfly database (http://redfly.ccr.buffalo.edu/; Gallo et al., 2011) several cis-regulating modules (CRMs) were found downstream of the site of interest, the best shot being the CRM esg_MLC2 localized 2642 bp downstream of esg (Halfon, 2014) and presumably enhancing the transcription rate of target genes in the midline glia, median neuroblast and its progeny (Long et al., 2014). If our insertion moves it away, we may expect the esg transcription rate in the developing nervous system of the embryo to be downregulated, in good accordance with our implied model. A violation of the esg_MLC2 regulation of esg transcription rate seems quite probable in the case of a large, $8,500 \mathrm{bp}$ insertion; however, its exact position relative to esg must be highly sensitive in order to admit the same effect as a small 32 bp insertion.

Other possible mechanisms involved in the downstream control of esg transcription, including tissue-specific transcription enhancement or repression and nucleosome shift or other types of chromatin remodeling, could be the focus of future studies.

\section{CONCLUSIONS}

To conclude, in our current study we planned to find out the following: (1) to corroborate the influence of the esg mutation on life span and describe its effects in more detail; (2) to find out whether the mutation affects esg expression and how gene expression should change in order to increase life span; (3) to understand whether the neuronal function of the gene is important for this. We demonstrated that the esg mutation affects gene transcription rate and proved that the decrease in esg expression is causally related to the life span increase. This crucial result for the first time demonstrated that esg is involved in life span control. We used the esg mutation to characterize effects of the gene on the life span of flies of different sexes and physiological statuses. We demonstrated that the esg mutation affects both male and female life span; that life span of females is affected less than lifespan of males; that life spans of unmated flies are affected more than life spans of mated flies; that aging is slowed down in mutant flies. We suggested that functional changes in tissues where esg is expressed (the nervous system, the gastrointestinal tract, testes) might be responsible for the effect on life span. As we are predominantly interested in unraveling the role of neuronal genes in life span control, we subjected the role of the esg function in the nervous system to a special study. For this purpose, effects of esg knockdown in the nervous system on life span were characterized. The results of these experiments allowed us to prove that the decrease in esg expression exclusively in the nervous system is sufficient to increases life span. By directly establishing the involvement of the neuronal function of esg in life span control, we have progressed toward achieving our main goal: to set a collection of genes encoding neuronal transcription factors involved in the life span control. This collection now includes shuttle craft (Roshina 
et al., 2014), Lim3 (Rybina et al., 2017) and esg and will be further used for analysis of molecular mechanisms underlying aging.

\section{AUTHOR CONTRIBUTIONS}

AS and NR have designed experiments; collected and interpreted data; critically revised the manuscript. AK have designed experiments; analyzed and interpreted data; critically revised the manuscript. EP have created the concept for the work and designed experiments; analyzed and interpreted data; drafted and critically revised the manuscript. All authors have approved the version to be published.

\section{FUNDING}

This work was supported by Russian Foundation for Basic Research grants \#15-04-05797-a and \#18-04-01127-a to EP.

\section{REFERENCES}

Alcedo, J., Flatt, T., and Pasyukova, E. G. (2013). Neuronal inputs and outputs of aging and longevity. Front. Genet. 4:71. doi: 10.3389/978-2-88 919-160-4

Ashraf, S. I., Hu, X., Roote, J., and Ip, Y. T. (1999). The mesoderm determinant Snail collaborates with related zinc-finger proteins to control Drosophila neurogenesis. EMBO J. 18, 6426-6438. doi: 10.1093/emboj/18. 22.6426

Ashraf, S. I., and Ip, Y. T. (2001). The Snail protein family regulates neuroblast expression of inscuteable and string, genes involved in asymmetry and cell division in Drosophila. Development 128, 4757-4767.

Bellen, H. J., Levis, R. W., Liao, G., He, Y., Carlson, J. W., Tsang, G., et al. (2004). The BDGP gene disruption project: single transposon insertions associated with $40 \%$ of Drosophila genes. Genetics 167, 761-781. doi: 10.1534/genetics.104.026427

Bhaskar, V., and Courey, A. J. (2002). The MADF-BESS domain factor Dip3 potentiates synergistic activation by Dorsal and Twist. Gene 299, 173-184. doi: 10.1016/S0378-1119(02)01058-2

Bhatt, D., and Ghosh, S. (2014). Regulation of the NF-кB-mediated transcription of inflammatory genes. Front. Immunol. 5:71. doi: 10.3389/fimmu.2014.00071

Cai, Y., Chia, W., and Yang, X. (2001). A family of snail-related zinc finger proteins regulates two distinct and parallel mechanisms that mediate Drosophila neuroblast asymmetric divisions. EMBO J. 20, 1704-1714. doi: 10.1093/emboj/20.7.1704

Cai, Y., Yu, F., Lin, S., Chia, W., and Yang, X. (2003). Apical complex genes control mitotic spindle geometry and relative size of daughter cells in Drosophila neuroblast and pI asymmetric divisions. Cell 112, 51-62. doi: 10.1016/S0092-8674(02)01170-4

Calo, E., and Wysocka, J. (2013). Modification of enhancer chromatin: what, how, and why? Mol. Cell. 49, 825-837. doi: 10.1016/j.molcel.2013.01.038

Carey, J. R. (2003). Longevity: The Biology and Demography of Life Span. Princeton, NT: Princeton University Press.

Dietzl, G., Chen, D., Schnorrer, F., Su, K. C., Barinova, Y., Fellner, M., et al. (2007). A genome-wide transgenic RNAi library for conditional gene inactivation in Drosophila. Nature 448, 151-156. doi: 10.1038/nature05954

Fuse, N., Matakatsu, H., Taniguchi, M., and Hayashi, S. (1999). Snail-type zinc finger proteins prevent neurogenesis in Scutoid and transgenic animals of Drosophila. Dev. Genes Evol. 209, 573-580. doi: 10.1007/s004270050291

Gallo, S. M., Gerrard, D. T., Miner, D., Simich, M., Des Soye, B., Bergman, C. M., et al. (2011). REDfly v3.0: toward a comprehensive database of transcriptional regulatory elements in Drosophila. Nucleic Acids Res. 39, D118-D123. doi: 10.1093/nar/gkq999

\section{ACKNOWLEDGMENTS}

Flies obtained from the Bloomington Drosophila Stock Center (NIH P40OD018537) were used in this study. We thank the TRiP at Harvard Medical School (NIH/NIGMS R01-GM084947) and Vienna Drosophila Resource Center (VDRC, www.vdrc.at) for providing the transgenic RNAi fly stocks used in this study. We are grateful to American Journal Experts (http://www.aje. $\mathrm{com} /$ ) for English editing services. We thank Trudy Mackay and members of her lab for fruitful collaboration that inspired this study. Special appreciation is owed to Dmitry Mukha for fruitful discussions, encouragement and support.

\section{SUPPLEMENTARY MATERIAL}

The Supplementary Material for this article can be found online at: https://www.frontiersin.org/articles/10.3389/fgene. 2018.00151/full\#supplementary-material

Garabedian, M. J., Shepherd, B. M., and Wensink, P. C. (1986). A tissue-specific transcription enhancer from the Drosophila yolk protein 1 gene. Cell 45, 859-867. doi: 10.1016/0092-8674(86)90560-X

Halfon, M. (2014). REDfly regulatory element database for Drosophila v5.3.1.

Hartl, M., Loschek, L. F., Stephan, D., Siju, K. P., Knappmeyer, C., and Kadow, I. C. (2011). A new prospero and microRNA-279 pathway restricts CO2 receptor neuron formation. J. Neurosci. 31, 15660-15673. doi: 10.1523/JNEUROSCI.2592-11.2011

Hekmat-Scafe, D. S., Dang, K. N., and Tanouye, M. A. (2005). Seizure suppression by gain-of-function escargot mutations. Genetics 169, 1477-1493. doi: 10.1534/genetics.104.036558

Hobert, O. (2011). Regulation of terminal differentiation programs in the nervous system. Annu. Rev. Cell Dev. Biol. 27, 681-696. doi: 10.1146/annurev-cellbio-092910-154226

Holden, P. R., Jones, P., and Brookfield, J. F. (1993). Evidence for a Wolbachia symbiont in Drosophila melanogaster. Genet. Res. 62, 23-29. doi: $10.1017 /$ S0016672300031529

Jasper, H. (2015). Exploring the physiology and pathology of aging in the intestine of Drosophila melanogaster. Invertebr. Reprod. Dev. 59(Suppl. 1), 51-58. doi: 10.1080/07924259.2014.963713

Jothi, R., Balaji, S., Wuster, A., Grochow, J. A., Gsponer, J., Przytycka, T. M., et al. (2009). Genomic analysis reveals a tight link between transcription factor dynamics and regulatory network architecture. Mol. Syst. Biol. 5:294. doi: $10.1038 / \mathrm{msb} .2009 .52$

Kim, H. J., Ahn, H. J., Lee, S., Kim, J. H., Park, J., Jeon, S. H., et al. (2015). Intrinsic dorsoventral patterning and extrinsic EGFR signaling genes control glial cell development in the Drosophila nervous system. Neuroscience 307, 242-252. doi: 10.1016/j.neuroscience.2015.08.049

Korzelius, J., Naumann, S. K., Loza-Coll, M. A., Chan, J. S., Dutta, D., Oberheim, J., et al. (2014). Escargot maintains stemness and suppresses differentiation in Drosophila intestinal stem cells. EMBO J. 33, 2967-2982. doi: $10.15252 /$ embj.201489072

Kwon, J. Y., Dahanukar, A., Weiss, L. A., and Carlson, J. R. (2007). The molecular basis of CO2 reception in Drosophila. Proc. Natl. Acad. Sci. U.S.A. 104, 3574-3578. doi: 10.1073/pnas.0700079104

Lapierre, L. R., Kumsta, C., Sandri, M., Andrea Ballabio, A., and Hansen, M. (2015). Transcriptional and epigenetic regulation of autophagy in aging. Autophagy 11, 867-880. doi: 10.1080/15548627.2015.1034410

Libert, S., Zwiener, J., Chu, X., Vanvoorhies, W., Roman, G., and Pletcher, S. D. (2007). Regulation of Drosophila life span by olfaction and food-derived odors. Science 315, 1133-1137. doi: 10.1126/science. 1136610

Long, S. K. R., Fulkerson, E., Breese, R., Hernandez, G., Davis, C., Melton, M. A., et al. (2014). A comparison of midline and tracheal 
gene regulation during Drosophila development. PLoS ONE 9:e85518. doi: 10.1371/journal.pone.0085518

Loza-Coll, M. A., and Jones, D. L. (2016). Simultaneous control of stemness and differentiation by the transcription factor Escargot in adult stem cells: how can we tease them apart? Fly 10, 53-59. doi: 10.1080/19336934.2016.11 76650

Loza-Coll, M. A., Southall, T. D., Sandall, S. L., Brand, A. H., and Jones, D. L. (2014). Regulation of Drosophila intestinal stem cell maintenance and differentiation by the transcription factor Escargot. EMBO J. 33, 2983-2996. doi: 10.15252/embj.201489050

Magwire, M. M., Yamamoto, A., Carbone, M. A., Roshina, N. V., Symonenko, A. V., Pasyukova, et al. (2010). Quantitative and molecular genetic analyses of mutations increasing Drosophila life span. PLoS Genet. 6:e1001037. doi: 10.1371/journal.pgen.1001037

Manzanares, M., Locascio, A., and Nieto, M. A. (2001). The increasing complexity of the Snail gene superfamily in metazoan evolution. Trends Genet. 17, 178-181. doi: 10.1016/S0168-9525(01)02232-6

McGraw, E. A., and O'Neill, S. L. (2004). Wolbachia pipientis: intracellular infection and pathogenesis in Drosophila. Curr. Opin. Microbiol. 7, 67-70. doi: 10.1016/j.mib.2003.12.003

Mukunda, L., Miazzi, F., Kaltofen, S., Hansson, B. S., and Wicher, D. (2014). Calmodulin modulates insect odorant receptor function. Cell Calcium. 55, 191-199. doi: 10.1016/j.ceca.2014.02.013

Pance, A. (2013). Tailoring the models of transcription. Int. J. Mol. Sci. 14, 7583-7597. doi: 10.3390/ijms14047583

Pasyukova, E. G., Roshina, N. V., and Mackay, T. F. (2004). Shuttle craft: a candidate quantitative trait gene for Drosophila lifespan. Aging Cell. 3, 297-307. doi: 10.1111/j.1474-9728.2004.00114.x

Pasyukova, E., Symonenko, A., Roshina, N., Trostnikov, M., Veselkina, E., and Rybina, O. (2015) "Neuronal genes and developmental neuronal pathways in Drosophila life span control," in Life Extension. Healthy Ageing and Longevity, Vol. 3, eds A. Vaiserman, A. Moskalev, and E. Pasyukova (Cham: Springer), $3-37$.

Poon, P. C., Kuo, T. H., Linford, N. J., Roman, G., and Pletcher, S. D. (2010). Carbon dioxide sensing modulates lifespan and physiology in Drosophila. PLoS Biol. 8:e1000356. doi: 10.1371/journal.pbio.1000356

Ramat, A., Audibert, A., Louvet-Vallée, S., Simon, F., Fichelson, P., and Gho, M. (2016). Escargot and Scratch regulate neural commitment by antagonizing Notch-activity in Drosophila sensory organs. Development 143, 3024-3034. doi: 10.1242/dev.134387

Reenan, R. A., Hanrahan, C. J., and Ganetzky, B. (2000). The mle (napts) RNA helicase mutation in Drosophila results in a splicing catastrophe of the para $\mathrm{Na}^{+}$channel transcript in a region of RNA editing. Neuron 25, 139-149. doi: 10.1016/S0896-6273(00)80878-8

Reenan, R. A., and Rogina, B. (2008). Acquired temperature-sensitive paralysis as a biomarker of declining neuronal function in aging Drosophila. Aging Cell. 7, 179-186. doi: 10.1111/j.1474-9726.2008.00368.x

Ridgel, A. L., and Ritzmann, R. E. (2005). Insights into age-related locomotor declines from studies of insects. Ageing Res. Rev. 4, 23-39. doi: 10.1016/j.arr.2004.08.002

Ringrose, L., and Paro, R. (2007). Polycomb/Trithorax response elements and epigenetic memory of cell identity. Development 134, 223-232. doi: 10.1242/dev.02723

Robertson, H. M., Preston, C. R., Phillis, R. W., Johnson-Schlitz, D. M., Benz, W. K., and Engels, W. R. (1988). A stable genomic source of P element transposase in Drosophila melanogaster. Genetics 118, 461-470.

Roshina, N. V., Symonenko, A. V., Krementsova, A. V., Trostnikov, M. V., and Pasyukova, E. G. (2014). Embryonic expression of shuttle craft, a Drosophila gene involved in neuron development, is associated with adult lifespan. Aging 6, 1076-1093. doi: 10.18632/aging.100712

Ruiz, M., Sanchez, D., Canal, I., Acebes, A., and Ganfornina, M. D. (2011). Sex-dependent modulation of longevity by two Drosophila homologues of human Apolipoprotein D, GLaz and NLaz. Exp. Gerontol. 46, 579-589. doi: 10.1016/j.exger.2011.02.014

Rybina, O. Y., and Pasyukova, E. G. (2010). A naturally occurring polymorphism at Drosophila melanogaster Lim3 locus, a homolog of human LHX3/4, affects Lim3 transcription and fly lifespan. PLoS ONE 5:e12621. doi: 10.1371/journal.pone.0012621

Rybina, O. Y., Sarantseva, S. V., Veselkina, E. R., Bolschakova, O. I., Symonenko, A. V., Krementsova, A. V., et al. (2017). Tissue-specific transcription of the neuronal gene Lim3 affects Drosophila melanogaster lifespan and locomotion. Biogerontology 18, 739-757. doi: 10.1007/s10522-017-9704-x

Sambrook, J., Maniatis, T., and Fritsch, E. F. (1989). Molecular Cloning: A Laboratory Manual. Cold Spring Harbor, NY: Cold Spring Harbor Laboratory.

Schaffner, W. (2015). Enhancers, enhancers - form their discovery to today's universe of transcription enhancers. Biol. Chem. 396, 311-327. doi: 10.1515/hsz-2014-0303

Schriner, S. E., Kuramada, S., Lopez, T. E., Truong, S., Pham, A., and Jafari, M. (2014). Extension of Drosophila lifespan by cinnamon through a sex-specific dependence on the insulin receptor substrate chico. Exp. Gerontol. 60, 220-230. doi: 10.1016/j.exger.2014.09.019

Schuettengruber, B., Chourrout, D., Vervoort, M., Leblanc, B., and Cavalli, G. (2007). Genome regulation by Polycomb and Trithorax proteins. Cell 128, 735-745. doi: 10.1016/j.cell.2007.02.009

Shaposhnikov, M., Proshkina, E., Shilova, L., Zhavoronkov, A., and Moskalev, A. (2015). Lifespan and stress resistance in Drosophila with overexpressed DNA repair genes. Sci. Rep. 5:15299. doi: 10.1038/srep15299

Skeath, J. B., and Thor, S. (2003). Genetic control of Drosophila nerve cord development. Curr. Opin. Neurobiol. 13, 8-15. doi: 10.1016/S0959-4388(03)00007-2

Spencer, C. C., Howell, C. E., Wright, A. R., and Promislow, D. E. (2003). Testing an 'aging gene' in long-lived Drosophila strains: increased longevity depends on sex and genetic background. Aging Cell 2, 123-130. doi: 10.1046/j.1474-9728.2003.00044.x

Spitz, F., and Furlong, E. E. M. (2012). Transcription factors: from enhancer binding to developmental control. Nat. Rev. Genet. 13, 613-626. doi: 10.1038/ $\operatorname{nrg} 3207$

The modENCODE Consortium, Roy, S., Ernst, J., Kharchenko, P. V., Kheradpour, P., Negre, N., et al. (2010). Identification of functional elements and regulatory circuits by Drosophila modENCODE. Science 330, 1787-1797. doi: $10.1126 /$ science.1198374

Tricoire, H., Battisti, V., Trannoy, S., Lasbleiz, C., Pret, A. M., and Monnier, V. (2009). The steroid hormone receptor EcR finely modulates Drosophila lifespan during adulthood in a sex-specific manner. Mech. Ageing Dev. 130, 547-552. doi: 10.1016/j.mad.2009.05.004

Voog, J., Sandall, S. L., Hime, G. R., Resende, L. P., Loza-Coll, M., Aslanian, A., et al. (2014). Escargot restricts niche cell to stem cell conversion in the Drosophila testis. Cell Rep. 7, 722-734. doi: 10.1016/j.celrep.2014.04.025

Wang, H. D., Kazemi-Esfariani, P., and Benzer, S. (2004). Multiple-stress analysis for isolation of Drosophila longevity genes. Proc. Natl. Acad. Sci. U.S.A. 101, 12610-12615. doi: 10.1073/pnas.0404648101

Werren, J. H., and Windsor, D. M. (2000). Wolbachia infection frequencies in insects: evidence of a global equilibrium? Proc. Biol. Sci. 267, 1277-1285. doi: $10.1098 / \mathrm{rspb} .2000 .1139$

Wilmoth, J. R., and Horiuchi, S. (1999). Rectangularization revisited: variability of age at death within human populations. Demography 36, 475-495. doi: $10.2307 / 2648085$

Wodarz, A., and Huttner, W. B. (2003). Asymmetric cell division during neurogenesis in Drosophila and vertebrates. Mech. Dev. 120, 1297-1309. doi: 10.1016/j.mod.2003.06.003

Yu, S., Yang, Y., Paik, D., Lee, E., and Park, J. J. (2015). Nmdmc overexpression extends Drosophila lifespan and reduces levels of mitochondrial reactive oxygen species. Biochem. Biophys. Res. Commun. 465, 845-850. doi: 10.1016/j.bbrc.2015.08.098

Zhong, Y., and Wu, C. F. (2004). Neuronal activity and adenylyl cyclase in environment-dependent plasticity of axonal outgrowth in Drosophila. J. Neurosci. 24, 1439-1445. doi: 10.1523/JNEUROSCI.074002.2004

Conflict of Interest Statement: The authors declare that the research was conducted in the absence of any commercial or financial relationships that could be construed as a potential conflict of interest.

Copyright (c) 2018 Symonenko, Roshina, Krementsova and Pasyukova. This is an open-access article distributed under the terms of the Creative Commons Attribution License (CC BY). The use, distribution or reproduction in other forums is permitted, provided the original author(s) and the copyright owner are credited and that the original publication in this journal is cited, in accordance with accepted academic practice. No use, distribution or reproduction is permitted which does not comply with these terms. 